CERN-EP/2001-051

$9^{\text {th }}$ July, 2001

\title{
Performance of a cluster of Multi-anode Photomultipliers equipped with lenses for use in a prototype RICH detector
}

\author{
E. Albrecht ${ }^{1}$, J. Baker ${ }^{2}$, G. Barber ${ }^{3}$, J. Bibby ${ }^{4}$, M. Calvi ${ }^{5}$, M. Charles ${ }^{4}$, A. Duane $^{3}$, S. Easo $^{6 a}$, \\ S. Eisenhardt ${ }^{7}$, L. Eklund ${ }^{1}$, M. French ${ }^{2}$, V. Gibson ${ }^{8}$, A. Halley ${ }^{6}$, R. Halsall ${ }^{2}$, N. Harnew ${ }^{4}$, \\ M.J.J. John ${ }^{3 b}$, S.G. Katvars ${ }^{8}$, J. Libby ${ }^{4 c}$, F. Muheim ${ }^{7}$, M. Paganoni ${ }^{5}$, A. Petrolini ${ }^{9}$, S. Playfer ${ }^{7}$, \\ D. Price ${ }^{3}$, J. Rademacker ${ }^{4}$, N. Smale ${ }^{4}$, S. Topp-Jorgenson ${ }^{4}$, D. Websdale ${ }^{3}$, G. Wilkinson ${ }^{4}$, \\ S.A. Wooton ${ }^{8}$
}

\footnotetext{
${ }^{1}$ CERN, European Organisation for Particle Physics, CH-1211 Geneva 23, Switzerland

${ }^{2}$ Rutherford Appleton Laboratory, Chilton, Didcot OX11 0QX, UK

${ }^{3}$ Imperial College of Science, Technology \& Medicine, Blackett Laboratory, Prince Consort Road, London SW7 2AZ, UK

${ }^{4}$ Department of Physics, University of Oxford, Keble Road, Oxford OX1 3RH, UK

${ }^{5}$ Dipartmento di Fisica, Univ. di Milano-Bicocca and INFN-MILANO, Piazza della Scienza 3, 20126, Milan, Italy

${ }^{6}$ University of Glasgow, Department of Physics, Glasgow G12 8QQ, UK

${ }^{7}$ Department of Physics and Astronomy, University of Edinburgh, JCMB King's Buildings, Mayfield Road, Edinburgh EH9 3JZ, UK

${ }^{8}$ University of Cambridge, Cavendish Laboratory, Madingley Road, Cambridge CB3 0HE, UK

${ }^{9}$ Dipartmento di Fisica, Università di Genova and INFN, Via Dodecaneso 33, 16146, Genova, Italy

${ }^{a}$ Now at the Rutherford Appleton Laboratory

${ }^{b}$ Now at Collège de France, Lab. de Physique Corpusculaire, IN2P3-CNRS, 75231 Paris Cedex 05, France

${ }^{c}$ Now at CERN
}

\begin{abstract}
A cluster of Multi-anode Photomultiplier Tubes (MaPMTs) equipped with focusing lenses in front of the tubes was tested in a prototype Ring Imaging Cerenkov detector in a charged particle beam. The readout electronics were capable of capturing the data at $40 \mathrm{MHz}$. The effects due to charged particles and magnetic field on the MaPMT performance were also studied. The results are used to evaluate the MaPMT as a possible photodetector for the LHCb RICH detectors.
\end{abstract}

Submitted to Nucl. Instr. and Meth. A. 


\section{Introduction}

This paper reports on the performance of Multianode Photomultiplier Tubes (MaPMTs) for the detection of Čerenkov light in a prototype Ring Imaging Čerenkov (RICH) detector. The MaPMTs are the back-up option ${ }^{1}$ for the photodetector to be used in the RICH detectors of the LHCb experiment [2] at the future Large Hadron Collider (LHC) at CERN. The performance studies presented here include, for the first time, those where the MaPMT data have been captured at $40 \mathrm{MHz}$, which is the bunch crossing rate of the LHC. A full description of the desired physics performance of $\mathrm{LHCb}$ and the subsequent requirements on the RICH detector can be found elsewhere [3]. A photodetector that meets these requirements must be both sensitive to single photons and spatially precise to a few millimetres. Furthermore, the device and accompanying electronics must have a fast enough response to be read out in $25 \mathrm{~ns}$.

A $3 \times 3$ array of MaPMTs has been tested using a RICH prototype in a beam at the CERN SPS facility. In order to reduce the losses of photons in the insensitive areas at the edges of the MaPMT, which corresponds to about $50 \%$ of the total area, the MaPMTs have been equipped with lenses. Pipelined readout electronics, compatible with the LHC 25 ns bunch crossing interval, have been tested for the first time on a RICH prototype. Also the effects of two potential hazards in a real detector environment have been investigated: the effect of charged particles traversing the MaPMT and the lenses, and the impact of different strength magnetic fields on the performance of the MaPMTs. For the magnetic field tests the possibility of shielding the MaPMTs against such fields was also studied.

The main aim of the tests was to demonstrate that the MaPMT is a viable photodetector for the LHCb RICH system. These tests aim to determine:

- the performance of the MaPMTs in an array, with and without lenses,

- the MaPMT's operation with pipelined readout electronics and,

\footnotetext{
${ }^{1}$ The baseline solution is the Pixel Hybrid Photo-diode Detector (HPD), studies of which are reported elsewhere [1].
}

- the functionality of the MaPMTs in a real detector environment.

Each of the above topics is reported below. In addition, laboratory tests of aspects of the MaPMT performance are presented.

The organisation of this paper is as follows. Section 2 contains a description of the hardware used in both the test beam and in the LED scanning facilities. The lenses and the pipelined electronic readout system are described in detail. The results of laboratory tests of the MaPMTs are reported in Section 3. In Section 4 the test beam results with respect to Čerenkov photon counting, with and without lenses, are presented. In addition, the effect of varying the angle of incidence between the incoming Cerenkov photons and the detector plane is investigated. The results are compared to a simulation of the RICH prototype. Section 5 and Section 6 give the results of the charged particle and magnetic field studies respectively. Finally, conclusions are given in Section 7.

\section{Hardware description}

\section{$2.1 \quad$ MaPMTs}

The Multi-anode Photomultiplier Tube (MaPMT) consists of an array of square anodes, each with its own metal dynode chain, incorporated into a single vacuum tube to amplify the photoelectrons emitted by the cathode. The densest pixelisation available, $8 \times 8$ pixels, provides the spatial resolution required for the $\mathrm{LHCb} \mathrm{RICH}$ detector. Figure 1 shows a schematic of the MaPMT. The dynode structure is divided into 64 channels of $2.0 \times 2.0 \mathrm{~mm}^{2}$ area, separated by $0.3 \mathrm{~mm}$ gaps.

The 64-pixel MaPMTs are commercially available and have been tested by LHCb in 1998 [1]. Since then the manufacturer, Hamamatsu, has provided some modifications which better match the LHCb RICH specifications. The MaPMT R760003-M64 ${ }^{2}$ described in this paper has a $0.8 \mathrm{~mm}$ thick UV-glass window with a semi-transparent bialkali

\footnotetext{
${ }^{2}$ With respect to its predecessor, the R5900-00-M64, the borosilicate window is replaced by a UV-glass window which increases the integrated quantum efficiency by $50 \%$. In addition, a flange of $1 \mathrm{~mm}$ size around the MaPMT is removed, thereby improving the packing fraction by $14 \%$.
} 


\begin{tabular}{|l|cccccccccccccc|}
\hline Dynode & PC & 1 & 2 & 3 & 4 & 5 & 6 & 7 & 8 & 9 & 10 & 11 & 12 & Anode \\
\hline$R$ in $\left[10^{2} \mathrm{k} \Omega\right.$ & $\mathbf{3}$ & 2 & 2 & 1 & 1 & 1 & 1 & 1 & 1 & 1 & 1 & 2 & 5 & \\
\hline
\end{tabular}

Table 1: Resistance chain used to provide the voltages at the photocathode (PC) and the dynodes.

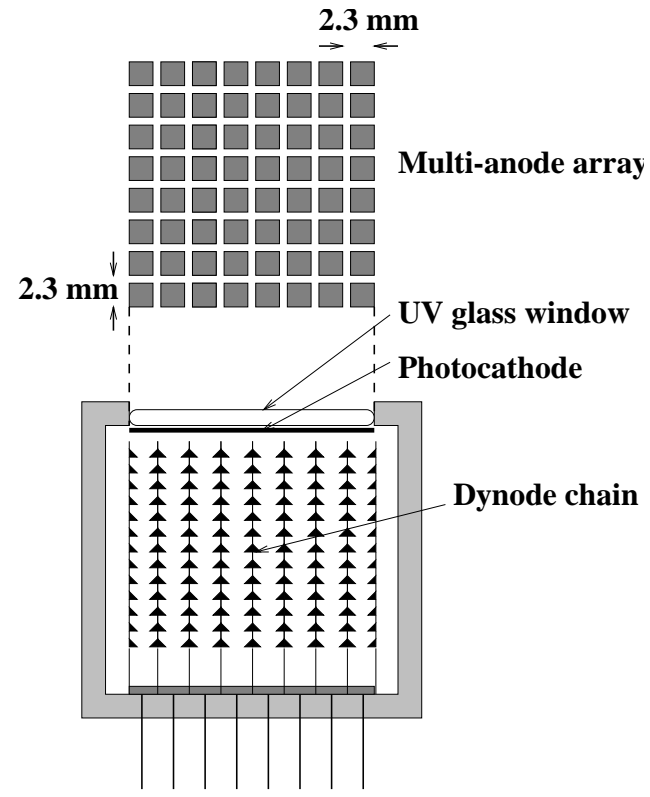

Figure 1: Sketch of the top and side view of an MaPMT.

photocathode deposited on the inside. The threshold for the light transmission through the UV-glass window is at a wavelength of $200 \mathrm{~nm}$. The quantum efficiency of the photocathode is plotted versus the wavelength of the photons in Figure 2. The quantum efficiency has a maximum of $22 \%$ at $380 \mathrm{~nm}$. For each pixel the photoelectrons are focused onto a 12-stage dynode chain (see Table 1) and multiplied through secondary emission. The mean gain of the MaPMT is about $3 \times 10^{5}$ when operated at a voltage of $800 \mathrm{~V}$.

Nine MaPMTs were purchased and preselected such that the average gain of the tubes varied by not more than a factor of two. A study of the variation of gain for the 64 pixel within one tube is presented in Section 3.

The geometrical coverage of the MaPMT, defined as the ratio of the sensitive photocathode area to the total tube area including the outer casing, is

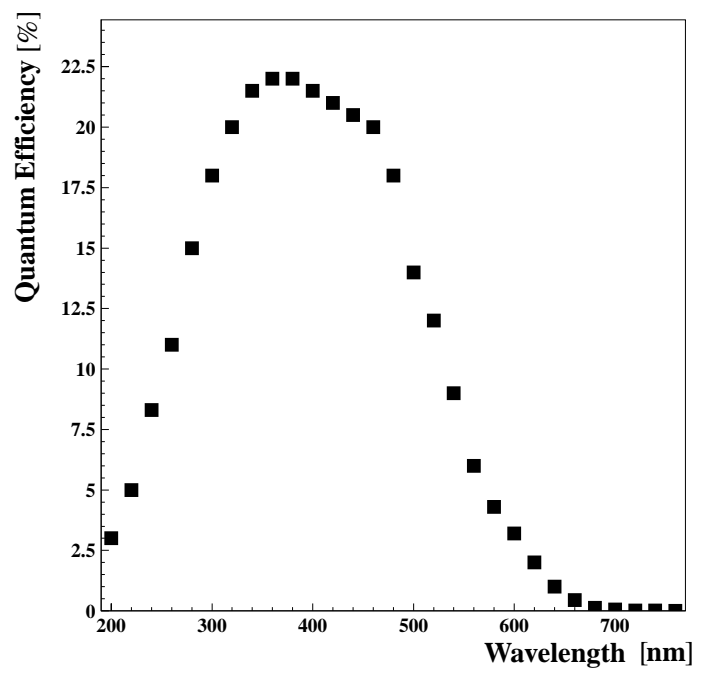

Figure 2: The quantum efficiency of the MaPMT R7600-03-M64 as a function of wavelength. The measurement was made by Hamamatsu.

only $48 \%$. This fraction can be increased by placing a single lens with one refracting and one flat surface in front of each MaPMT [4]. A single refracting surface with radius-of-curvature, $R$, has a focal length:

$$
f=\frac{R}{1-1 / n}
$$

where $n$ is the refractive index of the lens material. Figure 3 shows a schematic view of such a lens system in front of the close-packed photomultipliers. If the distance $d$ of the refracting surface to the photocathode is chosen to be equal to $R$, the demagnification factor is $(f-d) / f \approx 2 / 3$ for a quartz lens with $n=1.5$. Over the full aperture of the lens, light at normal incidence with respect to the photodetector plane is focused onto the photocathode, thus restoring full geometrical acceptance. The demagnification resulting from this lens configuration 


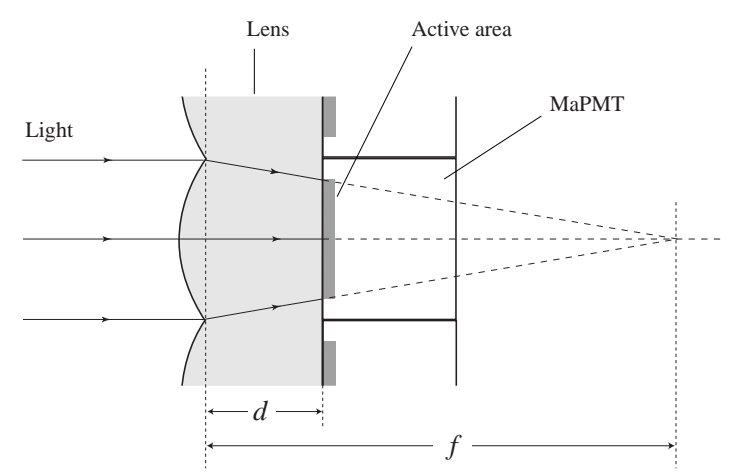

Figure 3: Schematic of a side view of the lens system, in front of the close-packed MaPMTs. The focusing of normally incident light is illustrated. The full aperture of the lens is focused onto the sensitive area of the MaPMT.

is illustrated in Figure 4. This demonstrates that the 'pin cushion' distortion is small compared to the pixel size. The focusing is also quite independent of the angle of incidence of light. Nine fused silica lenses, one for each MaPMT have been purchased ${ }^{3}$. The flat surface, which faces the photocathode is $26 \mathrm{~mm} \times 26 \mathrm{~mm}$, the radius of the spherical surface is $25 \mathrm{~mm}$ and the maximum height is $24 \mathrm{~mm}$.

\subsection{Experimental setup for beam tests}

The charged particle beam tests were carried out in the X7b beam at the CERN SPS. The beam was tuned to provide negative particles (95\% pions) with momenta $120 \pm 1 \mathrm{GeV} / \mathrm{c}$. The experimental setup included scintillation counters and a silicon pixel telescope to define and measure the direction of the charged particles, a vessel which contained $\mathrm{CF}_{4}$ gas radiator, a spherical mirror to focus the Cerenkov light and the MaPMT photodetector cluster. A schematic diagram of the Čerenkov vessel is shown in Figure 5. The components are briefly described below and a more complete description can be found in reference [5].

The beam telescope was comprised of 3 planes of silicon detectors, each segmented into a $22 \times 22$ ma-

\footnotetext{
${ }^{3}$ The lenses were machined and polished by Optical Works, Newquay, Cornwall, UK.
}

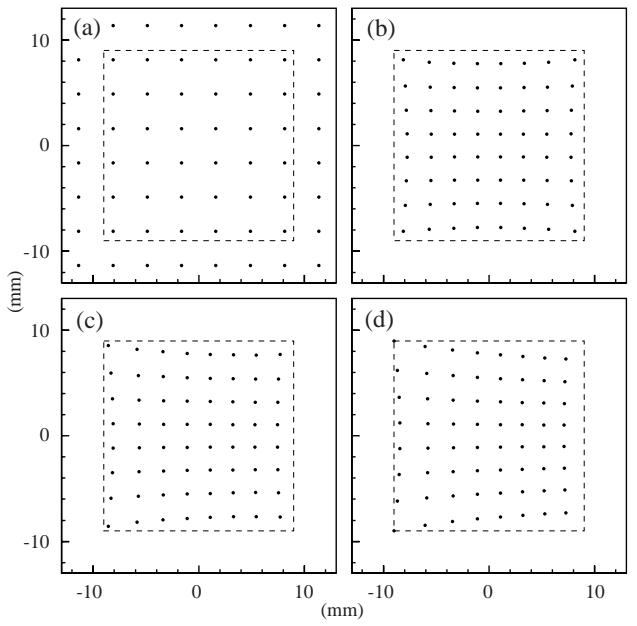

Figure 4: Impact points of a bundle of light rays incident on the entrance window of an MaPMT: (a) with no lens, (b-d) with a lens; (b) is for normally incident light, (c) and (d) are for an angle of incidence of $200 \mathrm{mrad}$ and $400 \mathrm{mrad}$ in the horizontal plane, respectively. The solid (dashed) line indicates the the total (sensitive) area of the tube.

trix of $1.3 \mathrm{~mm}$ square pixels. The pixels were read out using the Viking VA2 ASIC [6]. This is a 128channel amplifier-shaper-multiplexer chip, four of which are mounted on each telescope plane.

The Čerenkov vessel is a prototype geometry for the LHCb RICH 1 detector. Charged particles entered the radiator volume along a tube of $90 \mathrm{~mm}$ internal diameter and intersected the centre of a spherical aluminized-glass mirror which was tilted by $18^{\circ}$ to the beam axis. The plane of the MaPMT array was $1143 \mathrm{~mm}$ from the mirror centre. The mirror has a diameter of $112 \mathrm{~mm}$ and a focal length of $1117 \mathrm{~mm}$. Its reflectivity has been measured [1] to be $90 \%$ at $600 \mathrm{~nm}$, falling to $70 \%$ at $200 \mathrm{~nm}$. The mirror was mounted on micrometer screws, fixed to the back plate of the vessel. These allowed fine adjustments to precisely align the Cerenkov photons onto the photodetector array.

The Čerenkov radiator was a $\mathrm{CF}_{4}$ gas ${ }^{4}$ contained within a volume of length $1000 \mathrm{~mm}$ between the beam entrance window and the mirror. The gas was supplied at high pressure, via a recirculation system [1] to the vessel. The absolute pressure in

\footnotetext{
${ }^{4} \mathrm{CF}_{4}$ is the $\mathrm{LHCb} \mathrm{RICH} 2$ radiator.
} 


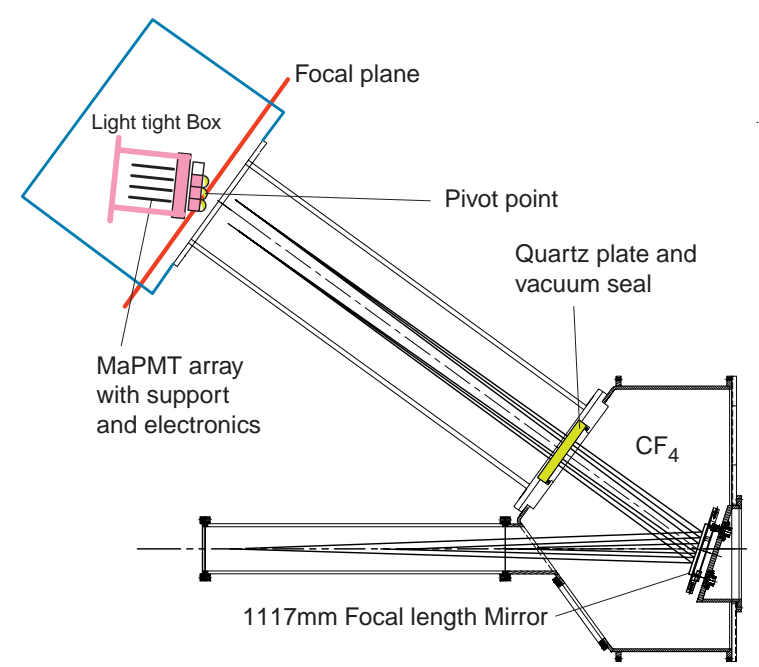

Figure 5: A schematic of the Čerenkov vessel.

the vessel could be set and stabilized by the system over a wide pressure range from 100-1200 mbar. Throughout data taking, gas pressure and temperature, water vapour and oxygen concentrations were monitored. These were used for correcting the $\mathrm{CF}_{4}$ refractive index, which has been parameterised as a function of wavelength at STP [7]. Data were collected with $\mathrm{CF}_{4}$ at a pressure of 700 mbar.

Between the mirror and the photodetectors the radiator vessel was sealed using a $25 \mathrm{~mm}$ thick fused silica plate. Initial tests, using a single MaPMT, verified that the loss of Cerenkov light resulting from transmission through this plate was $8 \%$, consistent with that expected due to reflection.

The fused silica lenses described in Section 2.1 were close-packed and mounted near to $(<$ $0.5 \mathrm{~mm}$ ), but not in optical contact with, the photocathode windows of the $3 \times 3$ array of MaPMTs. A micrometer screw allowed the position of the lens array to be displaced sideways by up to $10 \mathrm{~mm}$ with respect to the photocathodes. The overall photodetector assembly could be rotated with respect to the

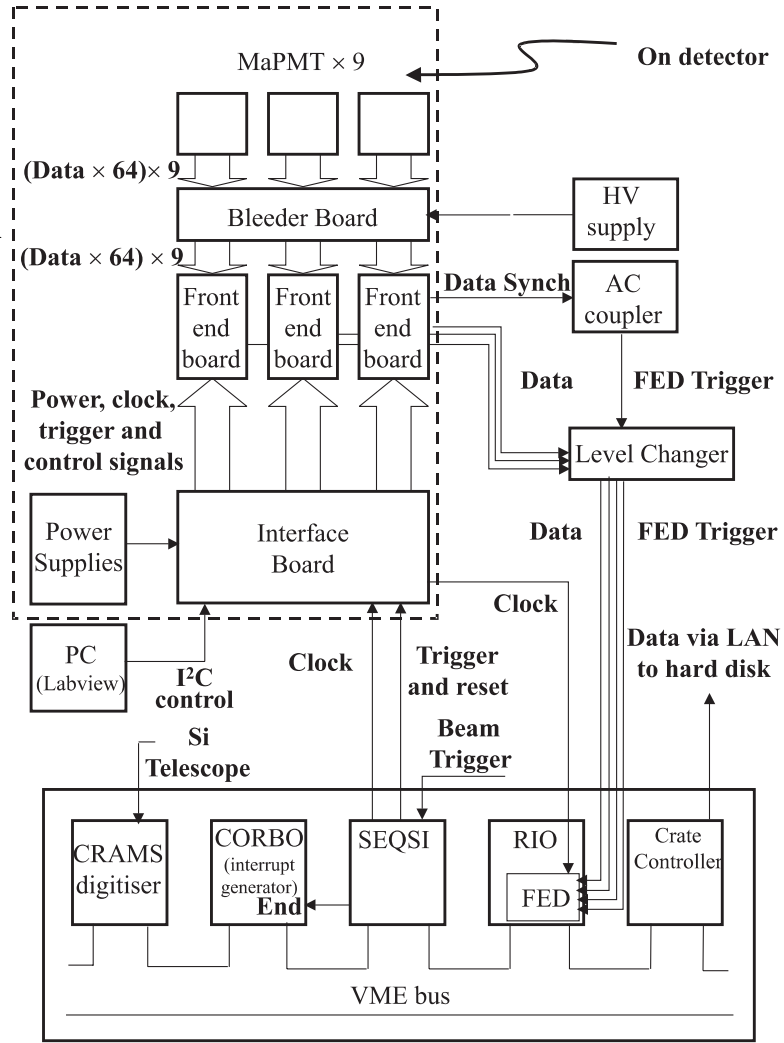

Figure 6: A schematic of the electronic readout and data acquisition systems. The components within the dashed box were those on the detector in the experimental area.

incident Čerenkov light. Unless otherwise specified data were collected at normal incidence.

\subsection{Readout electronics}

The tests involving individual MaPMTs were performed using a readout chain of CAMAC amplifiers and ADCs [1]. For the beam tests of the $3 \times 3$ array of MaPMTs a new pipelined electronic readout system was used, which is shown schematically in Figure 6. An overview of the system follows, in which several of the components (italicised) will be described in more detail.

Nine MaPMTs were mounted in a $3 \times 3$ array on a bleeder board unit, which supplied High Voltage (HV) to the MaPMTs and positioned them in the vessel. The output signal channels in the back plane of the bleeder board were coupled to 


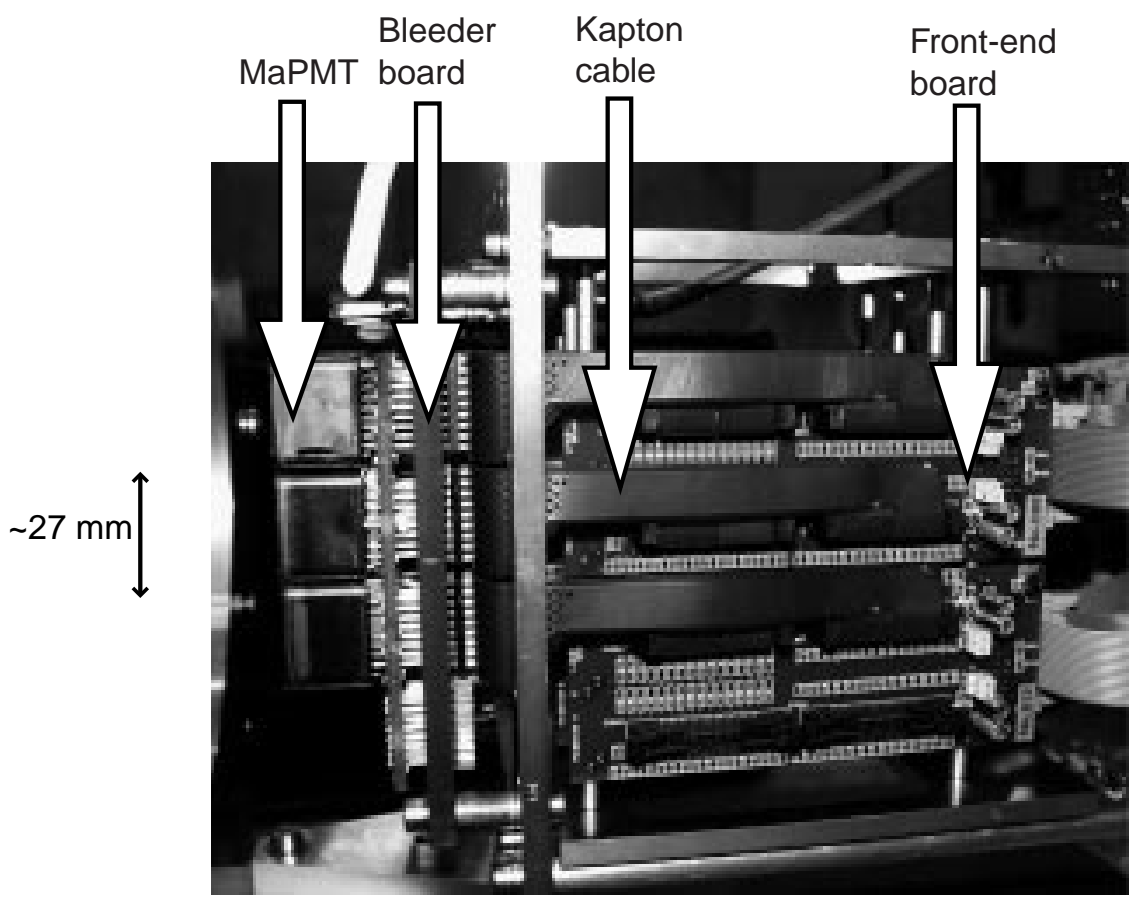

Figure 7: A photograph of the MaPMT array, bleeder board, front-end electronics board with APVm ASIC and AC-coupler network along with the mechanical support used in the test beam. The components described in Section 2.3 are indicated.

the front-end boards via kapton cables. Each frontend board multiplexed the analogue signals from one or two MaPMTs and was the carrier for the front-end ASIC (Application-Specific Integrated Circuit), the Analogue Pipeline Voltage (APVm) $[8,9,10]$. The APVm shaped, amplified, buffered and multiplexed the input signals. The frontend boards were then coupled to a single interface board, which fanned out the power, the trigger signals, the clock and the Philips $\mathrm{I}^{2} \mathrm{C}$ control signals [11] for the APVm. Figure 7 shows a photograph of the front-end electronics. The analogue pipeline signals from the APVm and a synchronous signal accompanying it were routed directly to the FrontEnd Digitiser (FED) and the rest of the data acquisition system, which is described in Section 2.4.

The bleeder board provided the mechanical support and dynode chain resistor network for up to 16 MaPMTs in a $4 \times 4$ array. The board also adapted the MaPMT anode feed-through pitch of the $16 \times 64$ data channels to the Pin Grid Array (PGA) pitch of the kapton cable. To achieve the minimum dead space between MaPMTs, while providing adequate $\mathrm{HV}$ isolation, careful layout of the board was required. In particular, the relative orientation of the MaPMTs and the size and layout of the dynode chain resistors minimised the voltage gradients between conductors. The bleeder board was constructed as two separate parts: the HV distribution board and the pitch adapter board. These were then assembled as a single unit using an array of soldered interconnect pins.

The kapton cables were used to couple the $8 \times 8$ output PGA for each tube, in the backplane of the bleeder board, to the front-end board. The kapton cables can be seen in Figure 7. The kapton cable from one MaPMT was coupled to two 40way SAMTEC ${ }^{5}$ connectors (64 data channels and 16 ground lines) on the front-end board.

The cables were flexible printed circuits with the tracks laid on one side of the kapton and a ground plane on the reverse. A cable was composed of

\footnotetext{
${ }^{5}$ Cable-to-board SFMC series.
} 


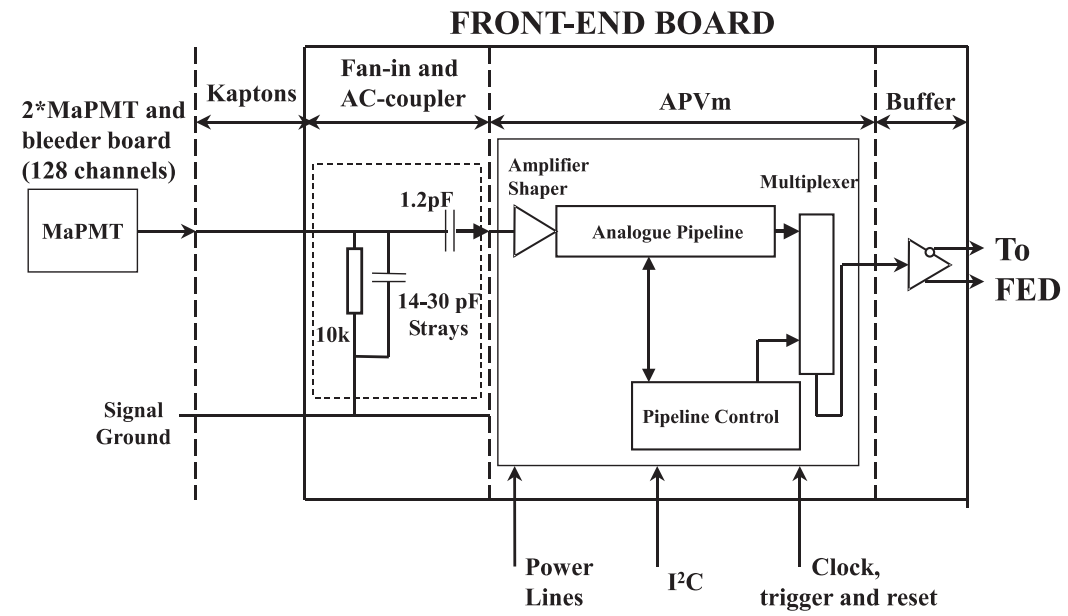

Figure 8: A schematic of the front-end board which carries the APVm ASIC.

4 strips of kapton; each strip coupled 2 columns of MaPMT output pins to half the channels of one SAMTEC connector. Each layer of kapton was coated with an insulator to isolate it from its neighbouring layers. To map the 4 layers to the two SAMTEC sockets on the front-end boards the pieces of kapton differed in length by up to a factor of two. These differences in the track lengths for the MaPMT data channels led to differing stray capacitance contributions to the attenuator network on the front-end board, which is described in Section 2.3. These stray capacitance values varied between 14 to $30 \mathrm{pF}$, which led to differences between channels in the attenuation factor of the AC-coupler described below.

The layout of the front-end board is given in Figure 8. The main components on the board are the AC-coupler network, which also acts as a fan-in, and the APVm ASIC. The APV ASICs were designed for use with silicon strip detectors or Micro-Strip Gas Chambers (MSGCs) which produce signals approximately 10 times smaller than those from an MaPMT. Therefore, the MaPMT signals had to be attenuated to be within the dynamic range of the APVm. This was achieved with an AC-coupler. The coupler was made from a gold plated ceramic base on which tracks were etched.

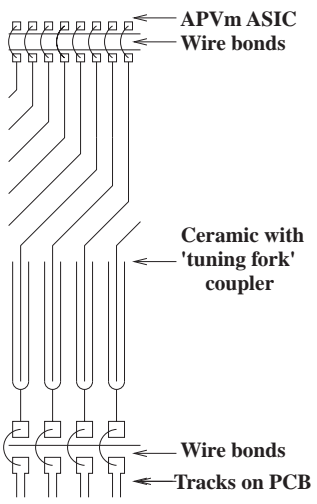

Figure 9: A section through the $\mathrm{AC}-$ coupler network.

The schematic of a section through the AC-coupler given in Figure 9 shows the 'tuning fork' capacitive coupling used.

The 128 active input signals were routed within a multi-layer Printed Circuit Board (PCB) from the 4 SAMTEC sockets to pads adjacent to one edge of the AC-coupler. The $128 \mathrm{PCB}$ pads were attached to the AC-coupler inputs with wire bonds pitched at $145 \mu \mathrm{m}$. The outputs from the AC-coupler were 


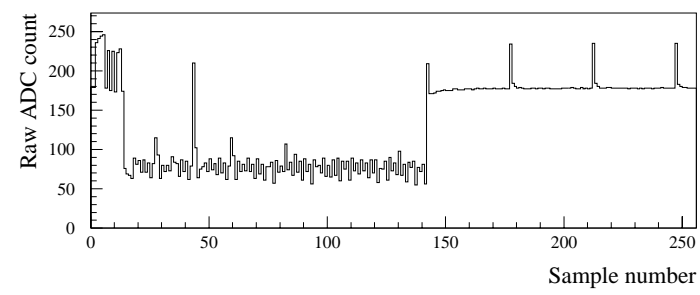

Figure 10: An example of the analogue output of the APVm ASIC. The header and pipeline address bits can be seen at the left-hand side of the frame. A signal can be seen at sample 43. Smaller signals are situated at \pm 16 samples either side of the principal peak; these are a consequence of crosstalk which is discussed in Section 4.

connected to the APVm ASIC inputs using wire bonds pitched at $60 \mu \mathrm{m}$. The front-end board also carried further components to perform three additional functions: $(i)$ buffers to drive the output data signals through $10 \mathrm{~m}$ of cable to the data acquisition system outside the experimental area, $(i i)$ components to set unique addresses for each frontend ASIC, so that the control voltages and currents could be set individually via an $\mathrm{I}^{2} \mathrm{C}$ control link and (iii) a switch to interrupt the power and thus reset the APVm.

The APVm [10] is one of the APV series [8, 9] of radiation-hard front-end ASICs which have been designed for the readout of the CMS inner detector [12]. The APVm has been fabricated using the Harris AVLSIRA bulk CMOS process [13]. These ASICs have been previously used in a beam environment for the readout of silicon-strip detectors [8].

In the test beam readout system the APVm ASIC, running at $40 \mathrm{MHz}$, sampled the 128 input analogue signals every $25 \mathrm{~ns}$. The signals were stored in a pipeline structure of 160 cells in depth, which allowed a maximum first-level trigger latency of $3.2 \mu \mathrm{s}$.

On arrival of a trigger to the APVm the data was tagged for readout. At a variable time later, which depended on the event's position in the pipeline the ASIC multiplexed the 128 signals into one analogue data output. The analogue output was cy- cled out of the ASIC at half the data-sampling rate $(20 \mathrm{MHz})$. Preceding the 128 samples there was a 12-bit header which contained the pipeline address at which the data were stored, and a flag to indicated if the ASIC was in error. The whole analogue data frame was $7 \mu \mathrm{s}$ in length. An example of the analogue output of the APVm is given in Figure 10. Further details of the pipeline control logic can be found in [10] and the references therein.

Synchronous to the data output there was a second output which changed level when a data frame was being read out, which is illustrated in the fifth line of the timing diagram given in Figure 11. This was used to trigger the data acquisition system as described in Section 2.4.

The APVm ASICs are not designed to meet the requirements of the $\mathrm{LHCb}$ trigger and readout architecture. In particular, the 1:128 multiplexing of the analogue signals at $20 \mathrm{MHz}$ precluded operation at the first-level trigger rate of $1 \mathrm{MHz}$ [14] and a signal return-to-zero within 25 ns was only possible in the de-convolution mode of APVm operation [8]. This requires 3 consecutive samples for the algorithm, and therefore prevents triggering on consecutive events. Nevertheless these tests provided an important indication that the use of MaPMTs will be compatible with the operation of the $\mathrm{LHCb} \mathrm{RICH}$ when equipped with appropriate front-end ASICs [15, 16], which are currently under development, the design of which addresses both of these issues.

The interface board fanned out the LVDS (Low Voltage Differential Signalling) trigger and clock signals, which were generated by the SEQSI [17] VME module, to the front-end boards. Furthermore, it distributed and filtered the $\pm 2 \mathrm{~V}$ and $\pm 5 \mathrm{~V}$ power supplies required to operate the APVm and the buffers on the front-end board respectively.

The control of the bias voltages, shaping currents, latency, operation and calibration modes of the $\mathrm{APVm}$, was done using the Philips $\mathrm{I}^{2} \mathrm{C}$ protocol. The control signals were generated on a $\mathrm{PC}$ using a LABView interface ${ }^{6}$ to drive an $\mathrm{I}^{2} \mathrm{C}$ PCIcard. An $\mathrm{I}^{2} \mathrm{C}$ bus extender ${ }^{7}$, placed on a separate card, was used to allow the signals to be driven over $10 \mathrm{~m}$ of cable into the experimental area. The $\mathrm{I}^{2} \mathrm{C}$ signals were fanned out to the front-end boards

\footnotetext{
${ }^{6}$ National Instruments.

${ }^{7}$ Philips P82B715.
} 


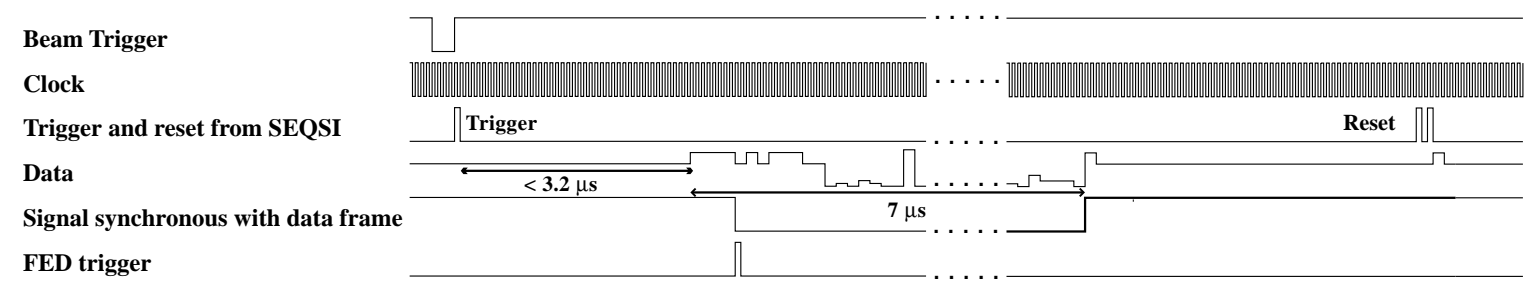

Figure 11: The timing diagram for the pipelined electronic readout and data acquisition system. The data frame begins a variable time up to $3.2 \mu \mathrm{s}$ after the beam trigger. The data frame is $7 \mu \mathrm{s}$ long.

by the interface board. The $\mathrm{I}^{2} \mathrm{C}$ signals generated at the PC may have been susceptible to ground loops and power surges related to the mains supply; therefore, to protect the front-end ASICs, the $\mathrm{I}^{2} \mathrm{C}$ signals were opto-coupled at the interface board.

\subsection{The data acquisition system}

The main components of the VME based data acquisition system are shown in Figure 6. Data were generated from three sources: the nine MaPMTs, the silicon beam telescope and the trigger scintillators. The event size is dominated by the nonzero-suppressed MaPMT data which amounted to about $1.5 \mathrm{kByte}$ per event. The acquisition system was capable of sustaining a trigger rate in excess of $1 \mathrm{kHz}$ for the $1.7 \mathrm{~s}$ duration of the SPS spill.

The beam trigger was formed from the coincidence between the four scintillators in the beam line. Any further beam triggers were gated out for the remainder of the readout cycle. The control of the front-end ASIC was performed using the outputs of the SEQSI programmable front-end control module. The timing diagram for the data acquisition system is shown in Figure 11. For times outside the readout cycle of the data acquisition system the SEQSI was in an 'idle loop', where no signals were sent to the front-end boards, apart from the continuous $40 \mathrm{MHz}$ clock. Once the NIM based trigger logic had made a positive decision a pulse (NIM type) was sent to the SEQSI to initiate the readout command sequence; the beam trigger signal is shown in the first line of the timing diagram. The SEQSI then produced a $25 \mathrm{~ns}$ wide LVDS pulse to trigger the front-end ASIC, which marked the event in the pipeline to be read out.

The critical aspect of the data acquisition sys- tem timing was the determination of the trigger logic latency. This was established by the simultaneous pulsed emission of light from LEDs positioned at the beam entrance to the RICH prototype vessel and within one of the scintillators used in the trigger ${ }^{8}$. This imitated the beam conditions of Čerenkov radiation in the RICH vessel and a charged particle causing scintillation light in the beam counters respectively. The latency of the ASICs was then varied in $25 \mathrm{~ns}$ steps, using the $\mathrm{I}^{2} \mathrm{C}$ control circuit, until the maximum signal was observed in the APVm output.

The six APVm ASICs each produced an analogue data output which was digitised using the Front-End Digitiser (FED) $[18,19]$. The FED is a PCI Mezzanine Card (PMC) which was affixed to a VME based motherboard and processor unit ${ }^{9}$. The FED PMC was a prototype module for the readout of the CMS inner tracker. The front-panel of the FED PMC has 8 analogue input data channels, a trigger and a clock input. The data from the front-end boards were both level shifted and amplified, to fall within the dynamic range of the Flash ADCs (FADCs) on the FED PMC, by a separate level changing board.

The $40 \mathrm{MHz}$ clock input was taken from the interface board; this was done to preserve the correct phase relationship between the clock and trigger input signals to which the FED PMC was sensitive. The trigger was required to be an LVDS signal of width less than $25 \mathrm{~ns}$. This was created from the signal synchronous to the data by $\mathrm{AC}$-coupling its front-edge and then converted this pulse signal to LVDS on the FED level changing board.

\footnotetext{
${ }^{8}$ For this calibration the trigger logic was reconfigured to require a signal in this scintillator alone.

${ }^{9}$ CES RIO, model No. 8061.
} 
Each analogue input to the FED was continually digitised by the 9-bit FADCs at $40 \mathrm{MHz}$. Once a trigger was received the data for 256 samples were stored in a Dual Port Memory (DPM). These samples included the 12 header bits and 128 channel samples ${ }^{10}$. The FED was programmed to act in a 'digital scope' mode, which means that each individual event was read out and no data compression was performed.

At the end of the readout sequence an APVm reset pattern was asserted by the SEQSI, on the APVm trigger output, which cleared the pipeline. Later, an 'end-of-readout' signal was generated at one of the SEQSI NIM output channels. This signal triggered the assertion of a VME interrupt using an interrupt generator module ${ }^{11}$. In response to this interrupt, a handler running in the readout processor then copied the data from the FED PMC event buffers into the processor's local memory, before resetting the readout sequence and then clearing the trigger veto.

Event data were accumulated in the readout processor's local memory during the SPS spill and were transferred via $100 \mathrm{MBit} / \mathrm{s}$ Ethernet connection to a mass storage device during the inter-spill gap.

\subsection{Monte Carlo simulation}

A detailed simulation program of the setup was developed, which is described in detail in [1]. The refractive index of the $\mathrm{CF}_{4}$ radiator as a function of the photon wavelength was obtained from the parametrization used in [7]. The values of the refractive index and the measured mirror reflectivity were identical to those given in [1].

The program also simulated the MaPMTs as described in Section 2.1, including the effects of lens focusing (Figure 3), the geometry of the detector surface, (Figure 1) and the quantum efficiency of the MaPMTs (Figure 2). A detailed electronics response (including the dynode chain) was not simulated. Instead the signal loss at the first dynode and below the threshold cut was estimated from the data. The average signal loss was subtracted MaPMT-by-MaPMT from the simulated photon

\footnotetext{
${ }^{10} \mathrm{As}$ the FED was operating at $40 \mathrm{MHz}$ and the data from the ASICs were being cycled out at $20 \mathrm{MHz}$, the FED was configured to take every other sample to reduce the event size by a factor of two.

${ }^{11}$ CORBO, CES RCB8047.
}

counts.

\subsection{LED scanning facilities}

Further tests of tubes and the electronic readout were performed using LED scanning facilities. Two such facilities were used. The general features of both facilities are described below and are shown schematically in Figure 12.

The MaPMTs were protected from extraneous light by a light-tight box. All cabling was brought into the box via feed-through connectors, which were isolated from the frame of the box to reduce ground loops. The tube was supported and clamped in a silicon resin-bonded fibre block. This provided insulation of the MaPMT casing, which was held at the cathode potential, to prevent current leakage across the window.

The light source used was a blue $470 \mathrm{~nm}$ LED with a maximum luminosity of $1000 \mathrm{mcd}$ and a view angle of $15^{\circ}$. The pulsing of the LED was performed using a FET circuit which provided a switching rate of $10 \mathrm{kHz}$ with a pulse duration of approximately $10 \mathrm{~ns}$. The LED was mounted externally and fed through to the dark box using a mono-mode optical fibre. This was done for two reasons: to restrict the light pulse to a narrow wavelength band with a Gaussian distribution and to minimise the spot size from the the end of the fibre by reducing aberrations. To reduce the LED spot size at the MaPMT window a sequence of lenses were used at the entry and the exit to the optical fibre. The width of the light spot from the fibre at the MaPMT window was $50 \mu \mathrm{m}$ or $100 \mu \mathrm{m}$ depending on the focusing system. These values were measured using a CCD camera.

The MaPMT and the fibre tip were both mounted on motorised stages. The stages could be positioned with a resolution better than $5 \mu \mathrm{m}$ which allowed precise scans over the MaPMT's acceptance. A stepper motor driver, interfaced to a $\mathrm{PC}$, was used to control the stages.

\section{LED scan results}

The response of the MaPMTs to single photons has been measured with the scanning facility described in Section 2.6. The photocathode of the MaPMT was operated at a voltage of $-900 \mathrm{~V}$. The LED in- 


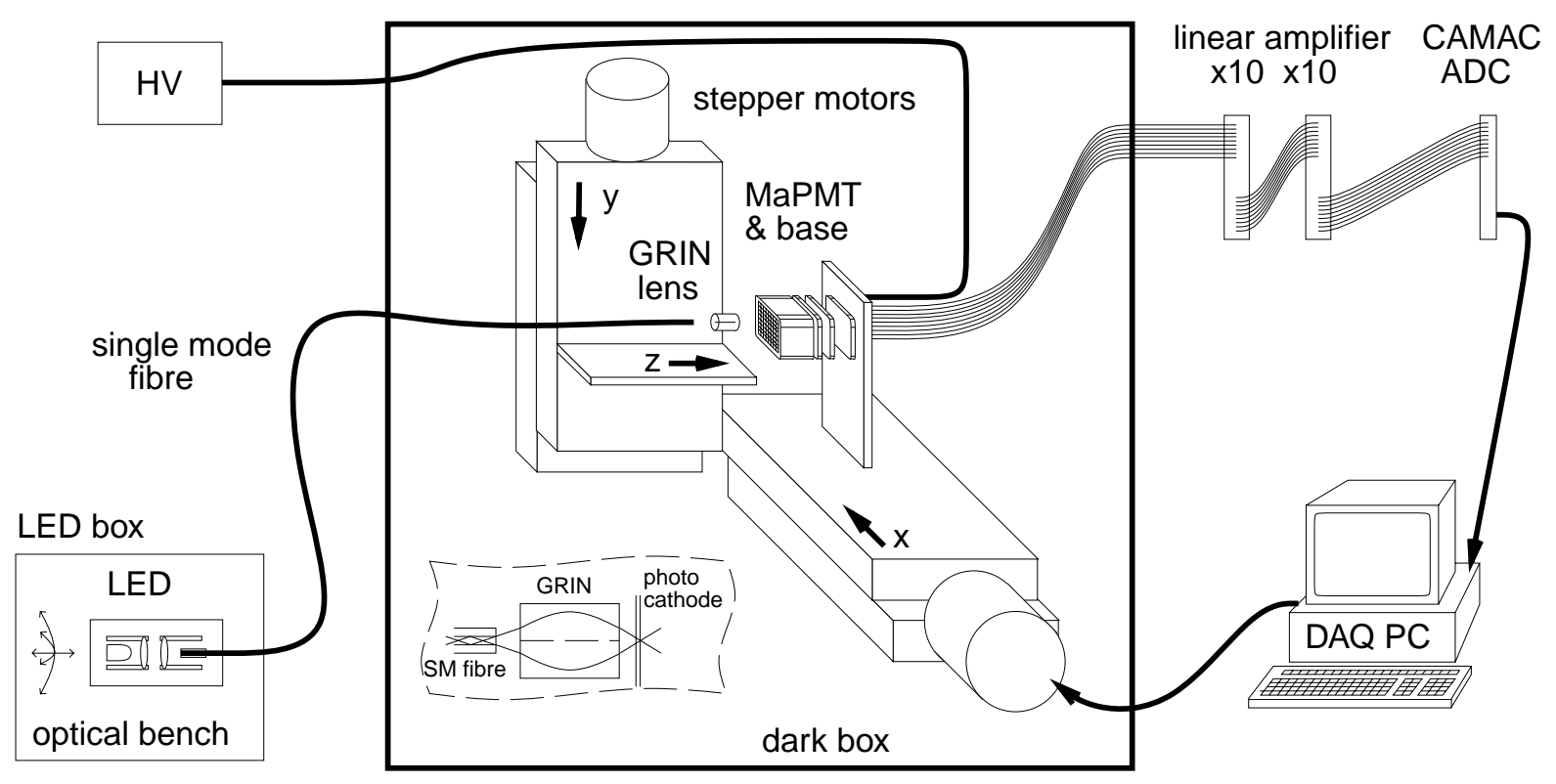

Figure 12: A schematic of one of the LED scanning facilities equipped with CAMAC readout electronics. Gradient Index (GRIN) lenses are used to focus the the LED at the entrance and exit (dashed inset) to the optical fibre.

tensity was set such that for $\sim 25 \%$ of the events at least one photoelectron was produced at the photocathode, within the $200 \mathrm{~ns}$ interval during which the signal was sampled. The events were recorded at a rate of $\sim 1000 \mathrm{~Hz}$. In Figure 13 a measured pulse-height spectrum is shown. The broad signal containing mostly one photoelectron and the narrow pedestal peak are clearly visible. The mean pulse-height, $s$, is approximately $90 \mathrm{ADC}$ counts above pedestals. The spectrum has been fitted by a function which allows a Gaussian shape for each photoelectron signal and for the pedestal peak; the number of photoelectrons in a pixel per event, $\lambda_{p e}$, has to follow a Poisson distribution. The sigma of the Gaussian signal shapes are constrained to be proportional to $\sqrt{\lambda_{p e}}$. The fit is superimposed on the data points. The sigma of the single photon signal, $\sigma_{s}$, is about $50 \mathrm{ADC}$ counts. The agreement between fit and data is good for large pulseheights which allows for reliable determination of $s, \sigma_{s}$ and $\lambda_{p e}$. The signal shape is non-Gaussian at small pulse-heights and as a consequence the fit fails to describe the data just above and below the pedestal peak. Furthermore, this region coun-

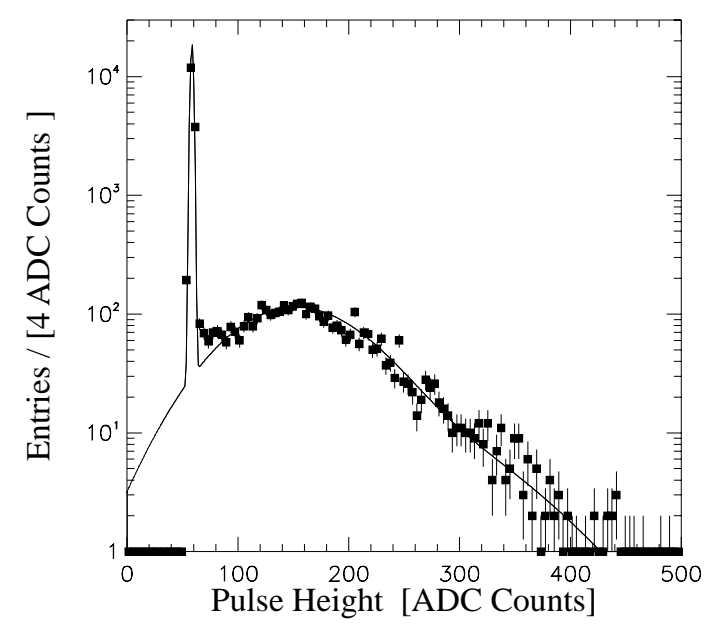

Figure 13: Single photon spectrum of an MaPMT pixel with a fit superimposed. 
(a)

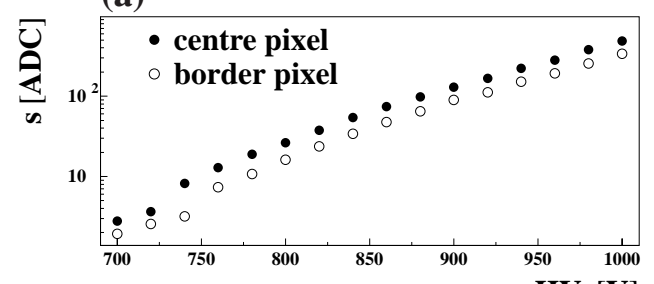

(b)

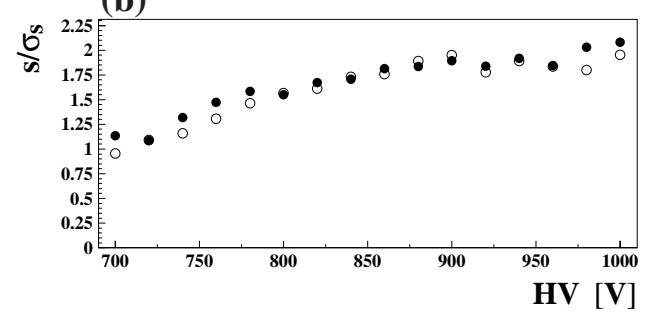

Figure 14: The measurements of (a) $s$ and (b) $s / \sigma_{s}$ as a function of high voltage. Measurements for pixels in the centre and at the border of the MaPMT are given.

tains a contribution from the photoelectric effect at the first dynode induced by photons transmitted through the photocathode window [20]. This effect has not been considered in the fit presented here.

The value of $s / \sigma_{s}$ gives an estimate of $\sqrt{g_{1}}$ to first order, where $g_{1}$ is the gain at the first dynode. For the pixels studied a lower limit of 3.7 for $g_{1}$ was found from $s / \sigma_{s}$. The probability that no multiplication occurs at the first dynode, $P_{1}$, is equal to $e^{-g_{1}}$. Therefore the gain measured corresponds to a value of $P_{1}$ of $2.5 \%$ or less. The signal to pedestal width ratio is $40: 1$.

The dependence of the gain of the MaPMT on different parameters has also been studied. The gain becomes larger with increasing negative high voltage applied to the photocathode. Figure 14 shows the measurements of $s$ and $s / \sigma_{s}$ as a function of the HV applied at the photocathode. The gain varies for the 64 different dynode chains within a tube. In Figure 15 the $s$ of all 64 channels of one tube is given. The gain varies by up to a factor of three with an RMS spread of about $30 \%$ about the mean value. Channels at the edge of the array have lower gain.
The edge pixels were investigated in more detail by scanning across the tubes in steps of $0.1 \mathrm{~mm}$. The measured gain as a function of the position of the light source across the tube is shown in the top of Figure 16. For the edge pixel the gain was not constant and dropped before the geometrical edge of the pixel. This was also reflected in the measurement of the average number of observed photons, $\lambda_{p e}$, as shown in the bottom of Figure 16. The collection efficiency, which is proportional to $\lambda_{p e}$, deteriorated towards the edge of the pixel. This reduced the overall efficiency of the MaPMT by a few percent. The pixel size is defined by the $50 \%$ efficiency points of a pixel and was found to be $2.1 \mathrm{~mm}$, which is a little larger than the $2.0 \mathrm{~mm}$ opening of the dynodes reported by the manufacturer.

\section{4 Čerenkov light detection}

Results obtained from measurements of Čerenkov rings using the MaPMT cluster equipped with lenses are presented in this section. The experimental setup and beam conditions are described in Section 2.2. The algorithms for correcting raw ADC counts for common-mode fluctuations and identifying crosstalk within the pipelined electronics are described in Sections 4.1 and 4.2. In Sections 4.34.5 , the measurement of the number of photoelectrons produced per charged particle is presented and the results compared to the simulation. Results are compared with and without lenses, and for different angles of incidence of Čerenkov radiation on the photodetector plane. All data used in the analysis were taken with a voltage of $-1000 \mathrm{~V}$ applied to the photocathode.

The numbering scheme used throughout this article for the MaPMTs and front-end boards is shown in Figure 17.

\subsection{Common mode correction}

In the first stage of analysis the data were corrected for common-mode fluctuations. These fluctuations were observed as time-dependent shifts in the pedestals of each channel, correlated throughout an MaPMT.

Common-mode fluctuations were corrected tube-by-tube. In a first iteration, the pedestal mean, $\mu$, and width, $\sigma$, were determined for each 


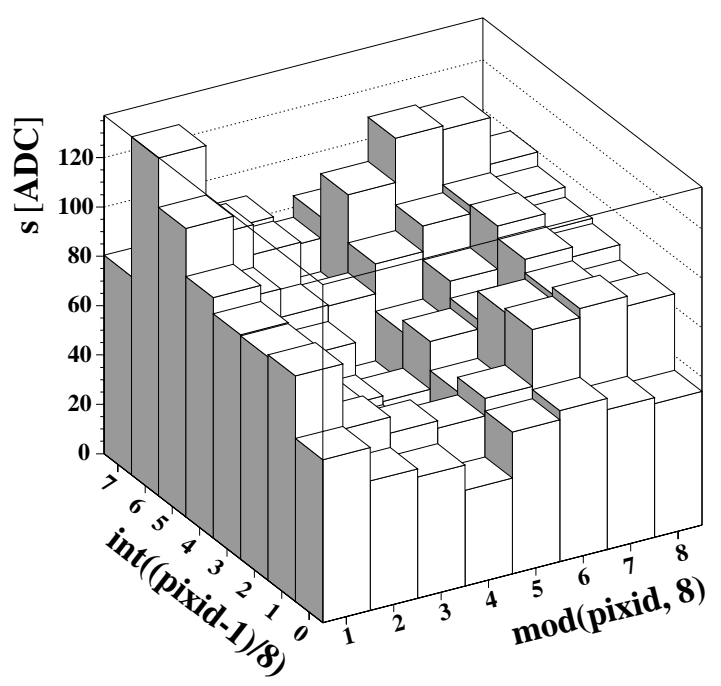

Figure 15: The measured value of $s$ for all 64 pixels of an MaPMT. The variable pixid in the axis labels is the pixel number in the range 1 to 64 .

(a)

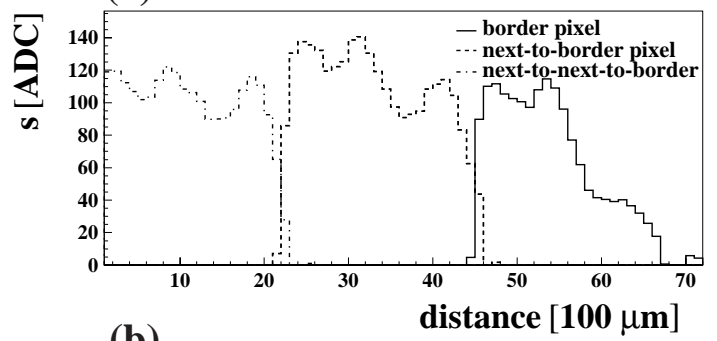

(b)

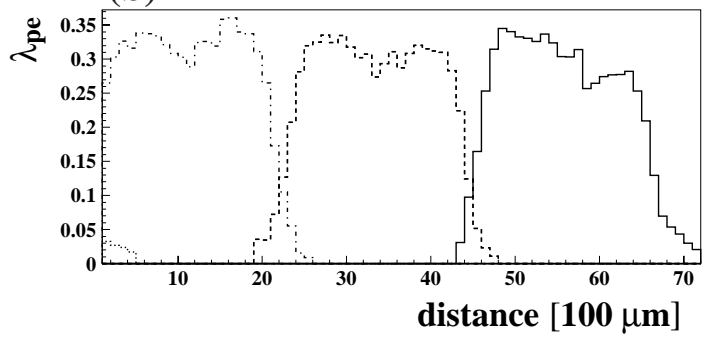

Figure 16: Scan across 3 MaPMT pixels. The measurements of (a) $s$ and (b) $\lambda_{p e}$ as a function of scanned position.

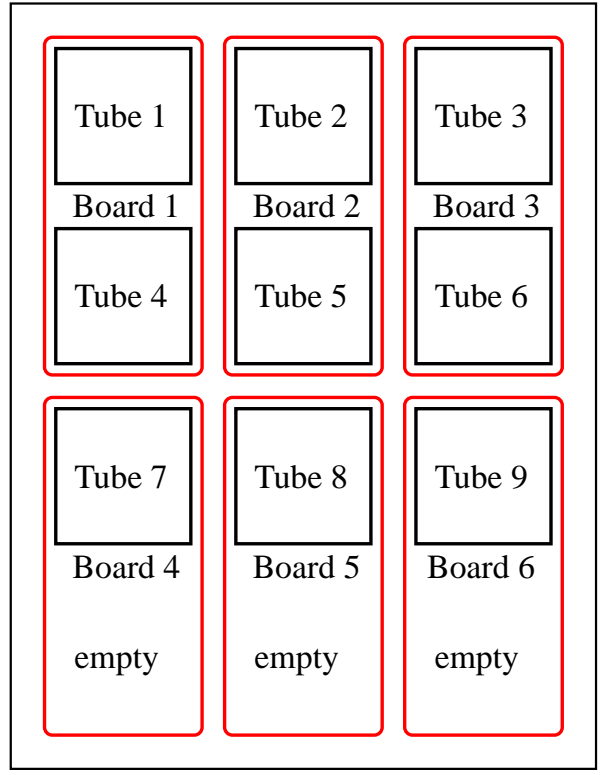

Figure 17: The physical positions, as seen by the incident Cerenkov photons, and the labels of the MaPMTs and the corresponding front-end boards.

pixel. The width after this stage overestimated the noise in the channel due to the common-mode contribution. The data were then reprocessed and pixels with a pulse-height above $\mu+3 \sigma$ were classified as a hit and were excluded. For the 64 or less pixels remaining without a hit in an individual event, the differences between the $\mathrm{ADC}$ values and the pedestal means, calculated in the first iteration, were averaged across a single tube. This gave the common-mode correction of that MaPMT for that event. This correction was subsequently subtracted from all pulse-heights for all pixels in an MaPMT.

Table 2 shows the common-mode correction for each tube. It is evident that the common-mode behaviour was very similar for tubes on the same front-end board. Laboratory tests where 0,1 or 2 tubes were connected to a front-end board confirmed that the common-mode fluctuations were associated with the front-end board and not to the tubes themselves. 


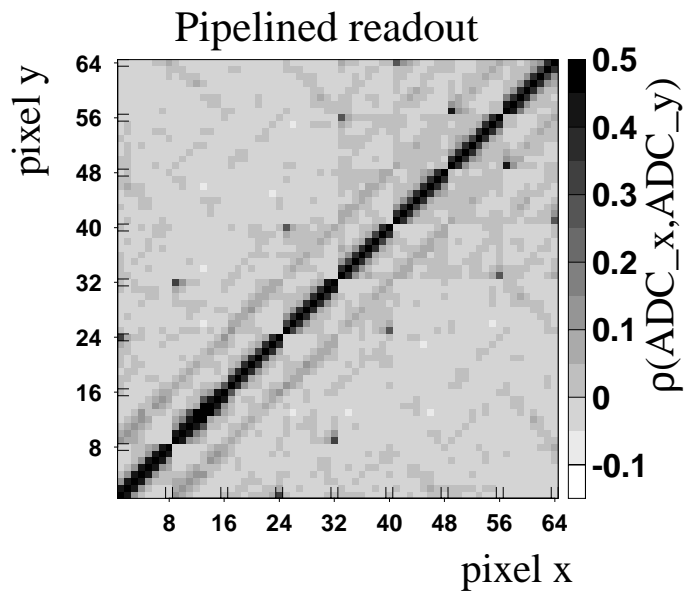

(a)

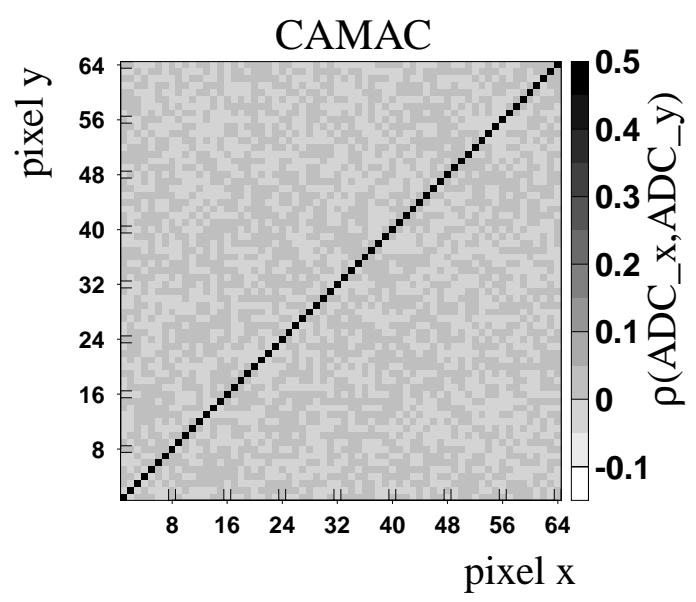

(b)

Figure 18: Correlation coefficients between the pulse-heights of pixels within one tube for the (a) pipelined and (b) CAMAC readout. For clarity the scale has been truncated to a maximum of 0.5. The diagonal has a correlation coefficient of 1 .

\begin{tabular}{|c|c||c||c|c|}
\hline \multirow{2}{*}{ Tube } & \multirow{2}{*}{ Board } & \multirow{2}{*}{$\begin{array}{c}\text { Common } \\
\text { Mode } \\
\text { correction }\end{array}$} & \multicolumn{2}{c|}{$\begin{array}{c}\text { Pedestal widths } \\
\text { [ADC counts }]\end{array}$} \\
\cline { 5 - 5 } & & & before & after \\
& & [ADC counts $]$ & & \\
\hline \hline 1 & 1 & 1.4 & 1.8 & 1.0 \\
4 & 1 & 1.3 & 1.7 & 1.0 \\
\hline 2 & 2 & 1.0 & 1.4 & 1.0 \\
5 & 2 & 1.0 & 1.4 & 1.0 \\
\hline 3 & 3 & 0.9 & 1.2 & 0.8 \\
6 & 3 & 0.8 & 1.1 & 0.8 \\
\hline 7 & 4 & 0.9 & 1.2 & 0.9 \\
\hline 8 & 5 & 0.8 & 1.2 & 0.8 \\
\hline 9 & 6 & 0.9 & 1.5 & 1.1 \\
\hline
\end{tabular}

Table 2: Widths of the pedestal peaks before and after common-mode correction for all tubes.

\subsection{Cross talk identification and cor- rection}

Crosstalk was identified using an LED to illuminate the MaPMT array uniformly with single photons.

Figure 18(a) shows the correlation coefficient, $\rho\left(\mathrm{ADC}_{x}, \mathrm{ADC}_{y}\right)$, between the pulse-heights of pixels $x, y$ within the same tube. The significant off- diagonal correlations indicate a large crosstalk between pixels. Figure $18(\mathrm{~b})$ shows $\rho\left(\mathrm{ADC}_{x}, \mathrm{ADC}_{y}\right)$ when reading out the MaPMT with CAMAC electronics. The absence of crosstalk in this latter case indicates the source of the crosstalk is located in the pipelined readout electronics chain and not within the tube.

To determine the nature of the crosstalk, the probability that a signal in pixel $x$ was induced by a hit in pixel $y$ was determined. Firstly a hit pixel was identified as having an ADC-value greater than $5 \sigma$ above the pedestal mean. In the case of a hit in both pixels $x$ and $y$, the pixel with the greater ADC-value was defined as the true photon hit; the other pixel hit was assumed to have been induced by crosstalk. The crosstalk probability is then defined as the ratio of the total number of of pixel $y$ induced hits in pixel $x$ to the total number of hits in pixel $x$. Genuine multi-photon events mean that this probability has a small offset from zero everywhere.

Figure 19 shows the crosstalk probabilities for one front-end board. It can be seen that the crosstalk extends across the board, hence is not contained within a single MaPMT. The crosstalk has a symmetric component, where pixel $y$ induces 
a signal in pixel $x$ and vice versa, and also an asymmetric component.

Three sources of crosstalk with differing strength were identified as originating from the readout electronics; these are listed in Table 3. The values for the asymmetric crosstalk varied widely between different ASICs used. Given the identified sources of crosstalk, a set of crosstalk partners which could induce a hit was associated to each pixel.

In subsequent photon-counting analyses using the pipelined electronics, the first step was the removal of crosstalk hits. For each hit pixel the crosstalk partners were also searched for a hit. The pixel under consideration was then discarded if any of its associated crosstalk partners had a hit with a greater pulse-height.

This method leads to a loss in efficiency due to the rejection of genuine hits in the crosstalk partners. Correction factors were applied pixel-bypixel to the data to correct for this effect. The corrections factors were dependent on the Cerenkov ring position on the MaPMT array. Integrated over the whole array, the total correction factor was $9 \%$.

An alternative crosstalk correction algorithm was also implemented for each pixel. This method involves the subtraction of a constant fraction of the pulse-height in pixel $y$ from the pulse-height measured in the crosstalk partner, pixel $x$. The fractions were determined from LED data. This method was used to correct the ADC spectra and to evaluate the systematic error due to the crosstalk correction.

Although significant crosstalk is observed in parts of the front-end readout system, in any future hardware iteration the crosstalk from the ACcoupler can be reduced by introducing a ground plane. Furthermore, in future iterations of the pipelined read out it is anticipated that the ASIC preamplifier gain will be better matched to the input signals. Therefore, an AC-coupler will no longer be necessary.

\subsection{Photon counting method}

Photon counting was performed for each pixel. Firstly, the mean $(\mu)$ and width $(\sigma)$ of the commonmode corrected ADC pedestal were found by fitting a Gaussian distribution. A threshold cut for each pixel was defined at $\mu+5 \sigma$; any $\mathrm{ADC}$ count above

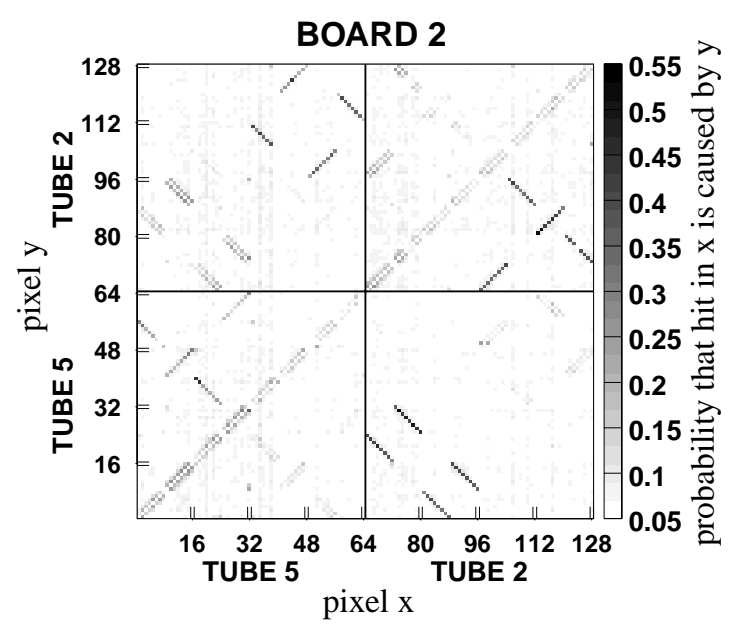

Figure 19: Crosstalk probabilities for board 9. The entries correspond to the probabilities that a signal in pixel $x$ (on the $x$-axis) was induced by a hit in pixel $y$ (on the $y$-axis).

\begin{tabular}{|c|c|}
\hline Source & $r$ \\
\hline \multicolumn{2}{|l|}{ Symmetric crosstalk } \\
\hline AC-coupler & 0.15 \\
\hline Kapton cables and PGA & 0.01 \\
\hline \multicolumn{2}{|l|}{ Asymmetric crosstalk } \\
\hline APVm ASIC & $0.01-0.33$ \\
\hline
\end{tabular}

Table 3: The sources of crosstalk and ratio of signal to crosstalk pulse--heights, $r$.

this threshold was considered as a hit. Crosstalk hits were removed as described previously.

The ratio of events, $f_{\text {hit }}$, with a hit in a given pixel to the total number of triggered events was calculated. $f_{\text {hit }}$ is related by Poisson statistics to the mean number of observed photons which give a hit per event, $\lambda_{\text {hit }}$, by:

$$
\lambda_{\text {hit }}=-\ln \left(1-f_{\text {hit }}\right) \text {. }
$$

This relationship assumes no signal loss. In reality there is loss arising from two sources. Firstly, the flow of electrons can terminate at a stage in the dynode chain, due to no secondary emission. This is dominated by the probability that a single 


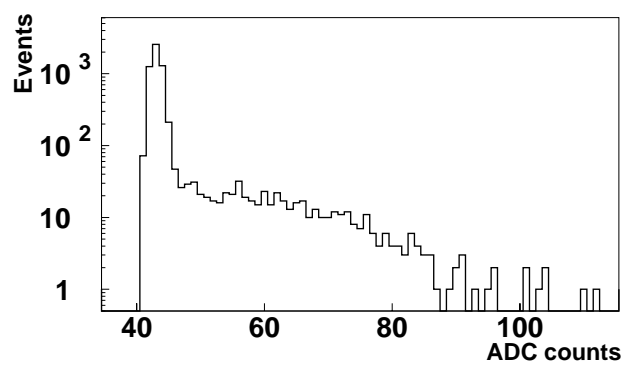

(a)

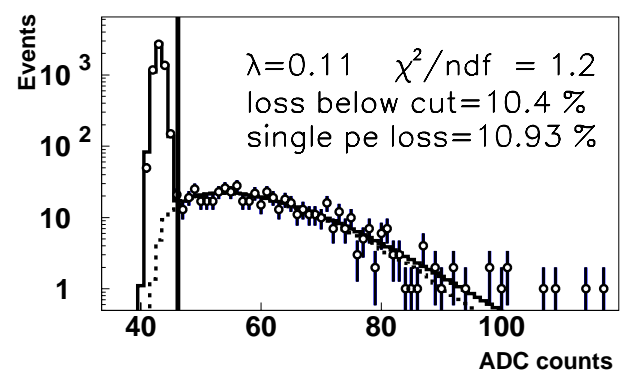

(b)

Figure 20: Spectra (a) before and (b) after correction. The plot of the spectra after correction has the fit superimposed (solid line). The single photoelectron part of the spectra is also shown (dashed line).

photoelectron arriving at the first dynode does not produce any secondaries, $P_{1}=e^{-g_{1}}$, where $g_{1}$ is the gain at the first dynode. The second source of signal loss is due to the pulse-height being below the threshold cut.

In making the comparison between data and simulation, a correction is made to the simulation for the relative fractions of signal loss, described in Section 4.4.

\subsection{Results from the MaPMT array}

The major correction to the photon counting is that associated to crosstalk; $\sim 45 \%$ of the pixel hits are from this source. An example of the crosstalk correction is given in Figure 20 which shows a typical

\begin{tabular}{|cccc|}
\hline Tube & $\begin{array}{c}\text { Loss } \\
\text { below cut } \\
(\%)\end{array}$ & $\begin{array}{c}\text { Gain } \\
\text { at 1st } \\
\text { dynode }\end{array}$ & $\begin{array}{c}\text { Total } \\
\text { loss } \\
(\%)\end{array}$ \\
\hline \hline 1 & $8 \pm 4$ & $3.2 \pm 0.5$ & $13 \pm 7$ \\
2 & $12 \pm 5$ & $3.9 \pm 0.4$ & $14 \pm 6$ \\
3 & $14 \pm 2$ & $3.2 \pm 0.4$ & $18 \pm 4$ \\
4 & $5 \pm 2$ & $4.0 \pm 0.4$ & $7 \pm 2$ \\
6 & $7 \pm 3$ & $3.8 \pm 0.8$ & $10 \pm 7$ \\
7 & $14 \pm 4$ & $3.2 \pm 0.4$ & $17 \pm 6$ \\
8 & $11 \pm 3$ & $3.4 \pm 0.5$ & $15 \pm 5$ \\
9 & $11 \pm 3$ & $3.7 \pm 0.7$ & $14 \pm 5$ \\
\hline
\end{tabular}

Table 4: Measurements of photon signal losses, averaged over pixels for each MaPMT.

pulse-height spectrum from Čerenkov light, before and after the crosstalk correction.

The corrected spectra were used to establish the signal loss in each pixel. The spectra were fitted with a function to describe the output of a photomultiplier tube. The fitting function describes the amplification of the dynode chain analytically as a series of Poissonian processes [21]. The five parameters returned by the fit are $\mu$ and $\sigma$ of the pedestal, the mean number of photoelectrons per pixel per event, $\lambda_{p e}, g_{1}$ and the conversion factor between the number of electrons at the end of the dynode chain and the ADC value. An example of a fit is shown in Figure 20(b). From the shape of the single photoelectron part of the function and the gain at the first dynode, both types of signal loss can be estimated. The excellent fit to the corrected data demonstrates that the crosstalk component has been successfully removed.

Table 4 shows the signal loss averaged over each MaPMT. The average total signal loss is $13 \%$, and the average values of the loss below threshold and $P_{1}$ are $10 \%$ and $3 \%$ respectively. The values in the table were used in the full simulation of the MaPMT array, described in Section 2.5.

LED runs were used to identify dead channels. Laboratory scans showed that the dead channels were associated with the readout electronics and not the MaPMTs. Also, noisy channels which contained a very large number of hits after the crosstalk correction were identified. Dead and noisy channels were masked in the photon counting analysis for both experimental and simulation data. Altogether 36 channels (6\% of the array) were re- 


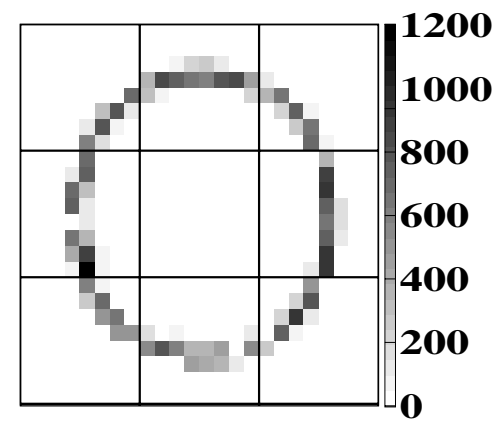

(a)

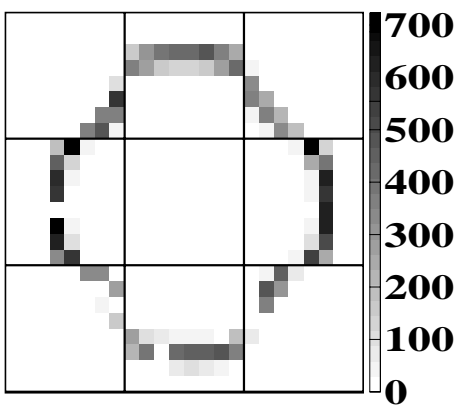

(b)

Figure 21: Čerenkov ring from a $1 \mathrm{~m} \mathrm{CF}_{4}$ radiator at a pressure of $700 \mathrm{mbar}$ integrated over $6 \mathrm{k}$ events, measured with the $3 \times 3$ array of MaPMTs (a) with, and (b) without quartz lenses mounted in front of the tubes. The insensitive areas between tubes are not shown and the active areas of adjacent tubes are plotted next to each other, hence the discontinuous ring without lenses in front of the MaPMTs.

moved; however, the number of masked channels on the Cerenkov ring with and without lenses was only 4 and 6 respectively.

The integrated signals for a run of 6000 events measured with the $3 \times 3$ array of MaPMTs with and without quartz lenses are shown in Figure 21. The Cerenkov rings are clearly visible, as is the effect of the lenses.

Finally, background hits were removed. Background arises from electronic and detector noise, and scattered Čerenkov light, assumed to be uniform over the array. The background was estimated by averaging the hits, MaPMT-by-MaPMT, over all pixels that were not lying on the Cerenkov ring.

In Table 5 the results on photon counting for the $3 \times 3$ array of MaPMTs are shown, with and without lenses. The mean numbers of hits per pixel per event, $\lambda_{\text {hits }}$, were summed over the whole array to give the total hits per event. The background contribution, integrated over the whole array, was subtracted to give the total number of photons per event, $N_{p e}$. The gain in $N_{\text {pe }}$ by employing the lenses is $55 \%$. The photon yield for the individual tubes is shown in Table 6; the results agree well with the simulation. From this it can be estimated that the relative quantum efficiency of the tubes varies by less than $\pm 10 \%$.

The large number of events (6000 per run) result in the statistical errors being $0.5 \%$. Systematic er-

\begin{tabular}{|c||ccc|}
\hline \multicolumn{1}{|c||}{} & \multicolumn{3}{c|}{$N_{\text {pe }}$ per tube } \\
\hline \hline \multirow{3}{*}{ Signal } & 0.62 & 1.11 & 0.65 \\
& 1.20 & 0.00 & 1.16 \\
& 0.66 & 0.90 & 0.66 \\
\hline \hline \multirow{3}{*}{ Simulation } & 0.56 & 1.10 & 0.68 \\
& 0.93 & 0.00 & 1.06 \\
& 0.58 & 0.91 & 0.67 \\
\hline
\end{tabular}

Table 6: Photon yields per event, $N_{\text {pe }}$, for the individual MaPMTs with lenses. The results are ordered as in the layout of Figure 17.

rors from several sources have been estimated. The major source of error is the crosstalk correction. This error was estimated to be $4.5 \%$ by comparing the results obtained with the alternative method of crosstalk removal, described in Section 4.2. An error of $1.2 \%$ was associated with the stability over the data-taking period. This was estimated by comparing the value of $N_{\text {pe }}$ over different runs. The total error on $N_{\text {pe }}$ is $4.7 \%$. There is an uncertainty on the simulation value of $N_{\text {pe }}$ of $2.6 \%$ due to the errors on the signal loss measurements. Within these errors the data on photon counting results are in reasonable agreement with simulation.

From the observed yield of photoelectrons, the figure of merit for a RICH detector, $N_{0}=$ 


\begin{tabular}{|c||c|c||c|}
\hline \multicolumn{1}{|c||}{} & \multicolumn{2}{c||}{ With lenses } & Without lenses \\
\hline & no correction & corrected & corrected \\
\hline \hline Data & 13.61 & 7.25 & 4.69 \\
Background & - & 0.29 & 0.21 \\
\hline Signal $\left[N_{p e}\right]$ & - & $6.96 \pm 0.33$ & $4.49 \pm 0.21$ \\
\hline \hline Simulated $\left[N_{p e}\right]$ & - & $6.49 \pm 0.17$ & $4.03 \pm 0.10$ \\
\hline
\end{tabular}

Table 5: Total photon yields per event for the $3 \times 3$ array of MaPMTs. For the case with lenses the yield before and after crosstalk correction is given.

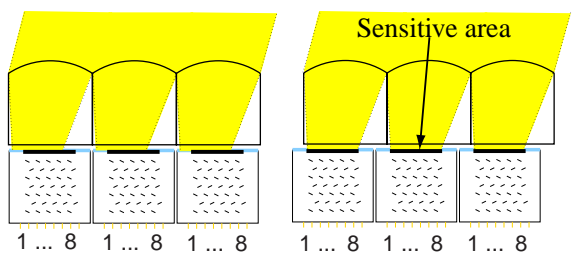

Figure 22: Recovering photons in a tilted MaPMT array by shifting the lenses relative to the MaPMTs.

$N_{\mathrm{pe}} /\left(\sin ^{2}\left(\theta_{C}\right) L\right)$, where $L$ is the length of the radiator $(100 \mathrm{~cm})$ and $\theta_{C}$ is the Črenkov angle, can be calculated. When extrapolated to atmospheric pressure, the value of $N_{0}$ is determined to be $157 \pm 10 \mathrm{~cm}^{-1}$. The measured value of $\theta_{C}$ was $25.5 \mathrm{mrad}$, compared to the expectation of $24.9 \mathrm{mrad}$. The average of these two numbers was used in the calculation of $N_{0}$, with the full difference taken as the error .

\subsection{Varying the angle of incidence}

The final LHCb RICH design may have a geometry where the Cerenkov light is not incident normal to the photodetector plane. Therefore, the performance of the array with lenses was tested for the case where the photons arrive at a varying angle $\alpha$ relative to the normal of the detector plane. LED runs were used for these tests.

Non-normal photon incidence leads to losses due to several sources. These include increased reflection at the surface of the lens, a reduction in effective area seen by photons from the mirror, which is proportional to $\cos \alpha$, and a shift in the image on the MaPMT photocathode. Provided the image is fully contained within the back surface of the

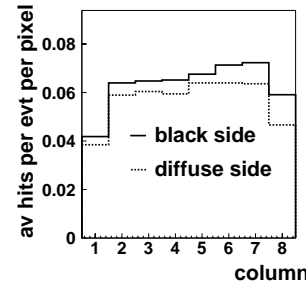

(a)

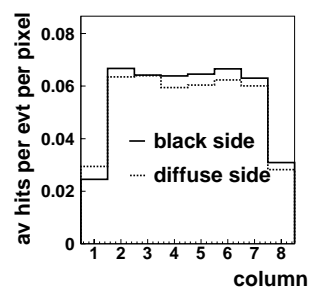

(c)

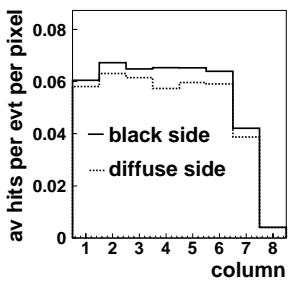

(b)

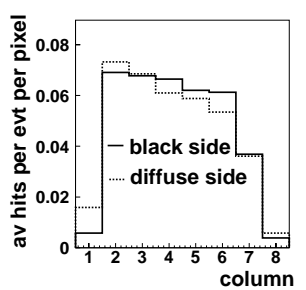

(d)
Figure 23: Average number of hits per pixel as a function of column number. Light was incident from the right (column 8) at angle $\alpha$. Four different configurations of incident light and lens displacement are shown: (a) $\alpha=0^{\circ}$, (b) $\alpha=20^{\circ}$ and displacement $=2.5 \mathrm{~mm}$, (c) $\alpha=20^{\circ}$ and displacement $=4.5 \mathrm{~mm}$, and (d) $\alpha=30^{\circ}$ and displacement $=5.8 \mathrm{~mm}$.

lens, which is in front of the MaPMT's photocathode, the loss due to the shift can be completely recovered by displacing the lens array relative to the MaPMTs. An ideal displacement is such that the photon image is fully contained within the sensitive area, as illustrated in Figure 22.

Columns of 8 pixels in each MaPMT were defined as shown in Figure 22. The hits per pixel per event averaged over a column is shown in Figure 23(a)(d), for different angles of incidence and lens displacements. It can be seen that ideal displacements 


\begin{tabular}{|c||c|c||c|c|}
\hline \multicolumn{1}{|c||}{$\begin{array}{c}\text { Angle of } \\
\text { incidence }\end{array}$} & \multicolumn{2}{c||}{ Blackened } & \multicolumn{2}{c|}{ Diffuse } \\
\cline { 2 - 5 } & Observed & Expected & Observed & Expected \\
\hline \hline $0^{\circ}$ & 4.05 & - & 3.64 & - \\
$20^{\circ}$ & 3.55 & 3.68 & 3.42 & 3.31 \\
$30^{\circ}$ & 2.98 & 2.93 & 2.98 & 2.64 \\
\hline
\end{tabular}

Table 7: Average number of hits per event per MaPMT at different angles. The expectations for $20^{\circ}$ and $30^{\circ}$ are normalised to the observed value at $0^{\circ}$.

of $4.5 \mathrm{~mm}$ and $5.8 \mathrm{~mm}$ were achieved for $\alpha$ of $20^{\circ}$ and $30^{\circ}$ respectively. A number of the lenses had blackened sides, while others had diffuse sides separated with white paper. The effect of reflection from the diffuse sides leads to a small increase for column 1, which can be seen in Figures 23 (c)-(d).

Table 7 summarises the results at different angles $\alpha$, where an ideal displacement in the lenses had been introduced; measurements are shown separately for lenses with blackened sides and lenses with diffuse sides. Results are compared with the expectation from the $0^{\circ}$ case, considering all the contributions to the loss of photons mentioned above.

For the $20^{\circ}$ case, the loss is dominated by the reduction in effective area, while for the $30^{\circ}$ case, a significant fraction of the lens image is outside the lens. The disagreement between expected and measured values for $30^{\circ}$ with diffuse sides is possibly due to some light being reflected back onto the photocathode from the lens sides, a contribution not included in the calculation of the expected value.

\section{Charged particle studies}

To study the effect of charged particles incident upon an MaPMT a single tube was placed, with its axis horizontal ${ }^{12}$, on a horizontal rotating platform in the beam line. Data were taken with $120 \mathrm{GeV} / \mathrm{c}$ pions traversing the MaPMT, with and without a quartz lens. Angles were scanned in the horizontal plane ranging from $0^{\circ}$, corresponding to charged particles incident on the MaPMT lens (or window), to $180^{\circ}$, corresponding to charged particles incident on the back of the MaPMT. The particle's impact

\footnotetext{
${ }^{12}$ The photodetector axis is defined as the normal direction to the photocathode, through its centre.
}

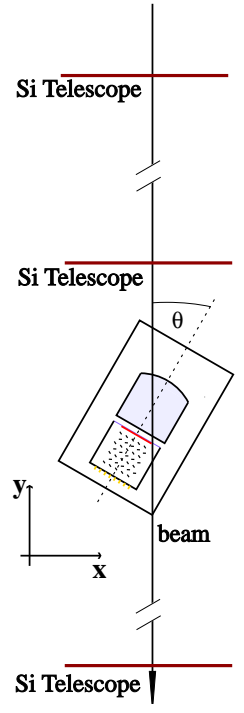

Figure 24: A schematic of the set-up for the charged particle studies, showing the silicon telescope, the angle definition and the centre of rotation (the centre of the lens).

point and trajectory were determined using the silicon telescope. For this analysis, only events with a single hit in each plane of the telescope were considered. The set-up and definition of the geometry are indicated in Figure 24.

The configuration was modelled in a dedicated simulation which included a full description of the optical properties of the MaPMT lens, window and the gap between the two. The properties of the photocathode, other than the net quantum efficiency, were assumed to be the same as those measured in [22].

Figure 25 shows the mean number of MaPMT hit pixels per charged particle as a function of the horizontal position of the particle in the silicon tele- 

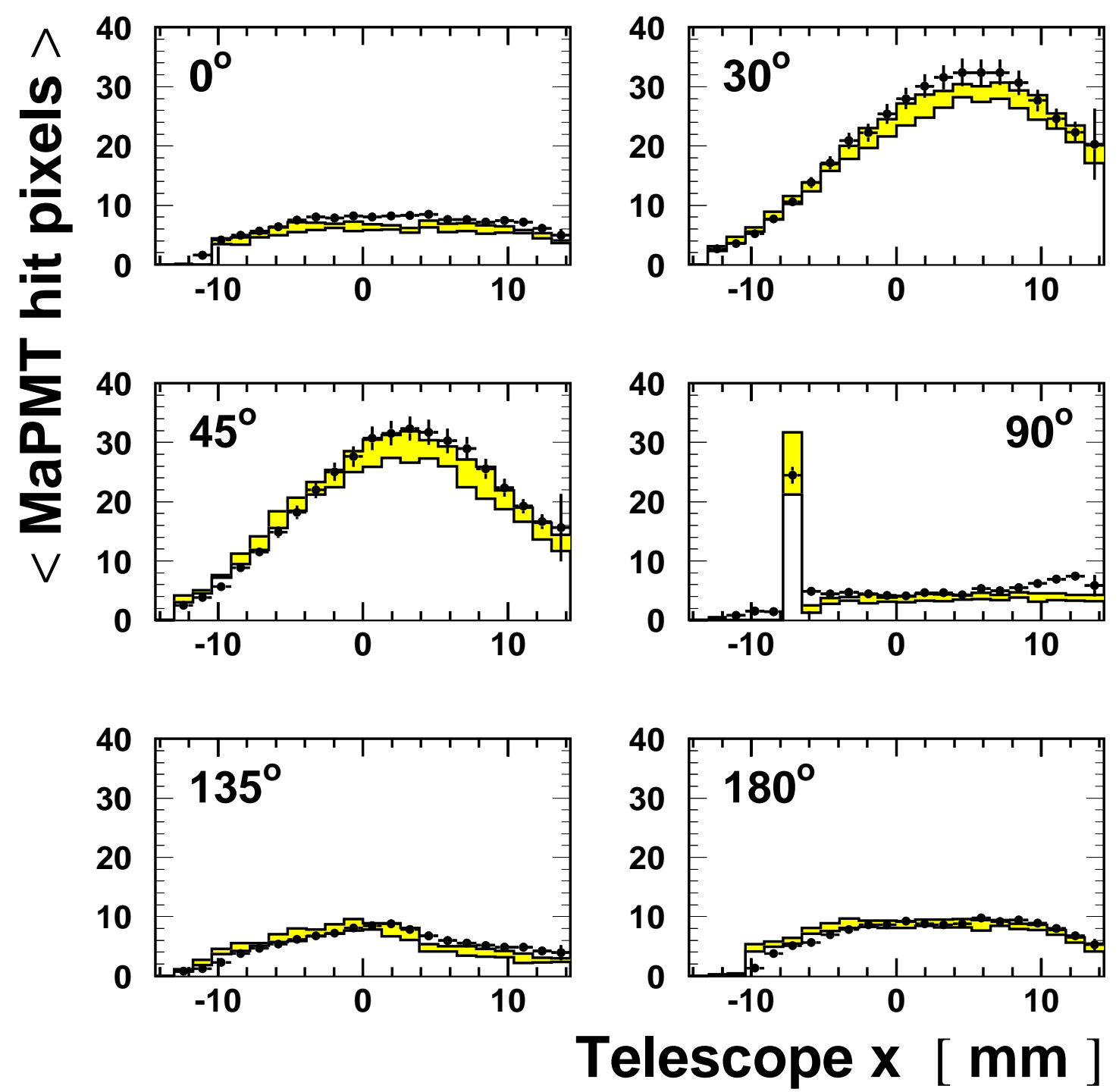

Figure 25: The mean number of hit pixels seen for incident charged particles for a MaPMT with lens. The results are plotted against the horizontal coordinate in the silicon telescope, and at different incident angles. Data are represented by the points and simulation by the solid line. The grey shading indicates the variations observed when the parameters of the optical modelling are varied within reasonable limits. 
scope, for different angles of beam incidence. In the vertical coordinate, the charged particle trajectory was limited to a band of $\pm 5.2 \mathrm{~mm}$ (4 silicon pixels) around the centre of the lens. Superimposed are the results from simulation, with the grey shading indicating the changes observed when the parameters in the optical modelling are varied within reasonable limits. The agreement between data and simulation is satisfactory.

The quartz lens provides a significant radiator length. However there is no optical coupling made between the lens and the window, and consequently many of the Cerenkov photons produced in the lens undergo internal reflection at this boundary. For small incoming particle angles fewer than 10 pixels are hit. At angles around $45^{\circ}$ many more of the photons produced in the lens are transmitted and penetrate the window, resulting in up to 30 hit pixels. At $90^{\circ}$ a sharp spike is seen for those particles which traverse the window parallel to the photocathode. At larger angles an average of less than 10 pixels are hit.

The MaPMT ADC spectra were fitted to establish how many photoelectrons contributed to each hit pixel. Significant variation was found, but certain pixels were observed to receive more than five photoelectrons. These results were consistent with the simulation.

With the flux of charged particles on the photodetectors expected in the $\mathrm{LHCb}$ experiment, such effects will not significantly degrade the performance of the RICH detectors [3].

A similar analysis was performed on data taken with a single MaPMT without a lens. Here about 5 hits per event were observed, with little dependence on the incident angle. In the special case where the particle traverses parallel to the photocathode, similar behaviour is seen to the data taken with a lens. These results are all well described by the simulation.

\section{$6 \quad$ Magnetic field studies}

The MaPMTs must operate in the fringe fields of the $\mathrm{LHCb}$ dipole magnet. The strength of these fringe fields is expected to be as high as $30 \mathrm{G}$ in the vicinity of the RICH photodetectors [2].

The sensitivity of the MaPMT to a magnetic field has been studied by placing a single tube within a

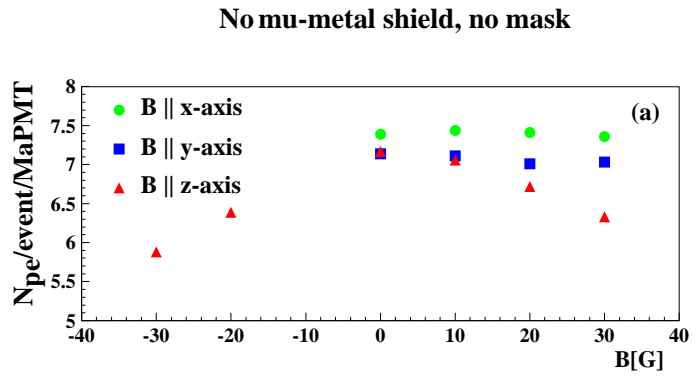

With mu-metal shield, B || z-axis

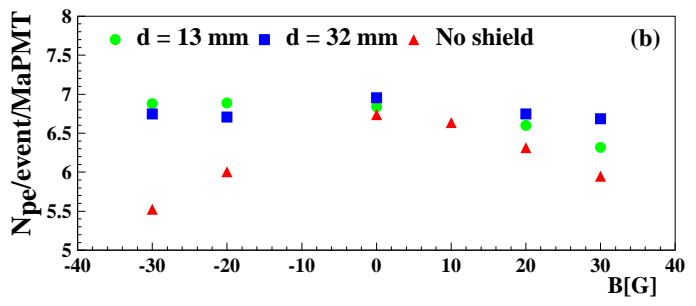

Figure 26: The number of observed photoelectrons from an LED source as a function of the magnetic field strength for (a) transverse $\left(B_{x}\right.$ and $\left.B_{y}\right)$ and longitudinal $\left(B_{z}\right)$ fields. In (b) the effect of shielding the MaPMT with a mu-metal tube at different $d$ (see text) is shown.

Helmholtz coil providing an axial magnetic fields of up to $30 \mathrm{G}$. Using an LED light source, the relative efficiency of a tube has been measured for magnetic fields transverse $\left(B_{x}\right.$ and $\left.B_{y}\right)$ and longitudinal $\left(B_{z}\right)$ to the photodetector axis. Figure 26(a) shows the measured number of photoelectrons as a function of the magnetic field strength for the three field orientations. This demonstrates that the MaPMT has little sensitivity to transverse magnetic fields up to $30 \mathrm{G}$. For longitudinal fields of $B_{z} \geq 10 \mathrm{G}$ the efficiency of the MaPMT deteriorates. This loss occurs mostly in the two edge rows, parallel to the $x$-axis where the focusing of the photoelectrons onto the first dynode is most sensitive to magnetic fields. At $B_{z}=30 \mathrm{G}$ the collection efficiency of the edge rows is reduced to $50 \%$ with respect to that at $B_{z}=0$ G.

The effect of shielding the MaPMT with a mumetal tube of square cross-section with a width 30 $\mathrm{mm}$, length $60 \mathrm{~mm}$ and wall thickness $0.9 \mathrm{~mm}$ was measured. The shield enclosed the tube and ex- 
tended beyond the MaPMT window face by a distance, $d$. Figure 26(b) shows the measured number of photoelectrons as a function of $B_{z}$ without and with the shield, for two values of $d(13 \mathrm{~mm}$ and $32 \mathrm{~mm}$ ). The mu-metal tube is effective in reducing the efficiency loss; with an extension of $32 \mathrm{~mm}$ or more there is no detectable loss in efficiency due to the $30 \mathrm{G}$ field.

\section{Summary and conclusions}

The performance of an MaPMT array equipped with lenses has been shown to agree well with expectations. The use of lenses to reduce the loss due to insensitive regions of the MaPMT has led to the expected improvement in the number of photoelectrons detected. The MaPMTs have been equipped with pipelined electronics to capture the data at 40 $\mathrm{MHz}$ which has performed well and Čerenkov rings have been clearly identified.

The effect of charged particles at different angles of incidence on an MaPMT equipped with a lens was found to agree well with expectations. Individual MaPMTs have been successfully operated in stray magnetic fields up to $30 \mathrm{G}$ with appropriate mu-metal shielding.

Finally, the MaPMT meets the photodetector requirements of the $\mathrm{LHCb} \mathrm{RICH}$ counters.

\section{Acknowledgements}

This work has benefited greatly from the technical support provided by our collegues at the institutes participating in this project. In particular the optical properties of the mirror and quartz window were measured by A. Braem and C. David, the bleeder board was laid out by C. Barham, the kapton cables were constructed by S. Greenwood, and the interface board was fabricated by R. Knott and H. Baughan. Valuable advice and assistance has been recieved from our colleagues in the LHCb collaboration, in particular O. Ullaland.

The CERN PS and SL divisions are thanked for providing the test beam and X7 facilities respectively. Finally, the UK Particle Physics and Astronomy Research Council (PPARC) and the Italian Istituto Nazionale Fisica Nucleare (INFN) for providing the financial support.

\section{References}

[1] E. Albrecht et al. Nucl. Instr. and Meth. A 456 (2001) 233.

[2] The LHCb Collaboration. LHCb Technical Proposal. CERN/LHCC/98-4.

[3] The LHCb Collaboration. LHCb RICH Technical Design Report. CERN/LHCC/2000-37.

[4] R. Forty. Use of lenses to increase the RICH photodetector coverage. LHCb/98/038.

[5] E. Albrecht et al. Nucl. Instr. and Meth. A 411 (1998) 249.

[6] O. Toker et al. Nucl. Instr. and Meth. A 340 (1994) 572.

[7] R. Abjean et al. Nucl. Instr and Meth. A 292 (1990) 593.

[8] MDM de Fez-Lazo et al. Nucl. Instr. and Meth. A 382 (1996) 533.

[9] M. Raymond et al. London 1997, Electronics for LHC Experiments. CERN/LHCC/97-60 (1997) 158.

[10] L.L. Jones et al. Rome 1998, Electronics for LHC Experiments. CERN/LHCC/98-36 (1998) 185.

[11] Philips Semiconductors. The $\mathrm{I}^{2} \mathrm{C}-\mathrm{Bus}$ Specification. Version 2.1, January 2000.

[12] The CMS Collaboration. The Tracker System Project Technical Design Report. CERN/LHCC/98-6.

[13] M. Raymond et al. Nucl. Instr. and Meth. A 351 (1994) 449.

[14] J. Christiansen. Requirements to the L0 frontend electronics. LHCb/99/029.

[15] N. van Bakel et al. Snowmass 1999, Electronics for LHC Experiments. CERN/LHCC/9833 (1999) 167.

[16] F. Anghinolfi et al. IEEE Trans. Nucl. Sci. 44 (1997) 298.

[17] M. Morrisey, Rutherford Appelton Laboratory. SEQSI Manual. Unpublished. 
[18] S.A. Baird et al. Snowmass 1999, Electronics for LHC Experiments. CERN/LHCC/98-33 (1999) 357.

[19] J. Coughlan, Rutherford Appelton Laboratory. CMS Front-End Driver PMC User Manual. Unpublished.

[20] I. Chikorov-Zorin et al. Nucl. Instr. and Meth. A 456 (2001) 310.

[21] J.Rademacker. Oxford University Nuclear Physics Preprint, OUNP-2001-2, to be submitted in Nucl. Instr. and Meth. A.

[22] M.E. Moorhead and N.W. Tanner. Nucl. Instr. and Meth. A 378 (1996) 162. 